\title{
Long-term effectiveness and drug survival of golimumab in patients affected by psoriatic arthritis with cutaneous involvement
}

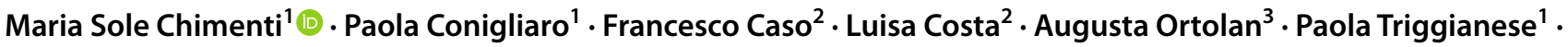 \\ Marco Tasso $^{2} \cdot$ Giulia Lavinia Fonti $^{1} \cdot$ Maria Grazia Lorenzin $^{3} \cdot$ Roberto Perricone $^{1} \cdot$ Roberta Ramonda $^{3}$
}

Received: 22 May 2021 / Revised: 19 July 2021 / Accepted: 28 July 2021 / Published online: 19 August 2021

(c) The Author(s) 2021

\begin{abstract}
Objectives To determine the effectiveness of golimumab (GLM) in improving joint, periarticular structures and cutaneous manifestations in patients with moderate to severe psoriatic arthritis (PsA) with cutaneous psoriasis in different real-life clinical settings and 48-month drug survival.

Methods Clinical and laboratory records were collected from PsA patients treated with GLM at baseline (T0) and after 6, $12,24,36$, and 48 months of treatment. Comparisons were performed using a paired $t$-test or Wilcoxon test. Drug survival rates were analyzed using Kaplan-Meier estimates. $p$ value $<0.05$ was considered statistically significant.

Results Data from 105 patients were collected. PsO occurred in $80 \%$ of patients and enthesitis in $78 \%$, peripheral and axial arthritis in $63.8 \%$ and $35.3 \%$, respectively, while erosions in $36.2 \%$. The main comorbidities were cardiovascular diseases (31.4\%) and metabolic syndrome (MetS) (19\%). A statistically significant improvement in articular and cutaneous psoriasis was registered at T48 of GLM-therapy in clinical (DAPSA $p<0.0001$; PASI $p<0.01$; BASDAI $p<0.0001$ ) and laboratory $(\mathrm{CRP}<0.05)$ indexes. Gender $(p=0.652)$, BMI $(p=0.655)$, smoking habit $(p=0.466)$, and line of treatment $(p=0.208)$ did not affect treatment efficacy nor persistence. At T48, $42 \%$ of patients discontinued GLM: the most frequent reason was an insufficient response or loss of efficacy (28.6\%).

Conclusion A 48-month GLM high drug persistence of PsA patients was observed in real-life, in patients presenting high disease activity, elevated prevalence of comorbidities, and more than one line of treatment at baseline. Patients' characteristics as gender, smoke, BMI, different lines of treatment, and concomitant methotrexate treatment affected treatment persistence, making GLM effective and safe in moderate-severe PsA in a long-term real-life setting.
\end{abstract}

\section{Key Points}

- Golimumab was effective in psoriatic arthritis, including both musculoskeletal and cutaneous manifestations.

- Golimumab effectiveness and drug survival were not affected by comorbidities and patient-related characteristics.

- The 4-year drug survival curves confirm the efficacy and safety of golimumab in psoriatic arthritis patients in a real-life setting.

Keywords Drug survival $\cdot$ Golimumab $\cdot$ Long-term effectiveness $\cdot$ Psoriasis $\cdot$ Psoriatic arthritis $\cdot$ Real-life

Maria Sole Chimenti and Paola Conigliaro are equally contributed to this work.

Maria Sole Chimenti

maria.sole.chimenti@uniroma2.it

1 Rheumatology, Allergology and Clinical Immunology, Department of Medicina Dei Sistemi, University of Rome Tor Vergata, Via Montpellier 1, Rome, Italy

2 Rheumatology Unit, Department of Clinical Medicine and Surgery, University Federico II, Naples, Italy

3 Rheumatology Unit, Department of Medicine DIMED, University of Padova, Via Giustiniani 2, Padua, Italy

\section{Introduction}

Psoriatic arthritis (PsA) is a chronic inflammatory disease, belonging to the group of spondyloarthritis ( $\mathrm{SpA})$, typically associated with psoriasis ( $\mathrm{PsO}$ ) and characterized by the presence of both articular and periarticular structures involvement [1]. The prevalence of $\mathrm{PsO}$ ranges from 2 to $3 \%$ in the general population, and PsA may affect 10-30\% of $\mathrm{PsO}$ patients [2]. Joint involvement in PsA is a potentially debilitating disease: patients may present arthritis to both small and large peripheral joints, enthesitis, and the axial 
disease, leading to progressive erosive arthritis and severe functional impairment in more than half of the patients [3]. PsA pathogenesis is only partially understood and still remains to be completely clarified. The presence and the overexpression of cytokines such as tumor necrosis factor (TNF)- $\alpha$, interleukin (IL)-17, and IL-23, plays a role in the pathogenic ways linking PsA and PsO [4]. The use of TNF inhibitors (TNFi) approximately two decades ago has dramatically improved PsA treatment. However, several unmet needs concerning the management of both joint and skin manifestations still remained [5]. Randomized-placebo controlled clinical trials involving TNFi have shown excellent results for PsA and PsO treatment. Etanercept, infliximab, adalimumab, golimumab (GLM), and certolizumab have been demonstrated to be effective in PsA and in distinctive aspects of the disease, as skin disease, peripheral arthritis, enthesitis, and dactylitis. Moreover, their effectiveness was demonstrated in improving quality of life and work ability and in reducing radiographic progression [6]. GLM is a TNFi approved by the European Medicines Agency (EMA) and the US Food and Drug Administration (FDA) for the treatment of active rheumatoid arthritis, active PsA, active ankylosing spondylitis, severe non-radiographic axial SpA, polyarticular juvenile idiopathic arthritis, and moderate to severe active ulcerative colitis [7]. GLM is a fully human monoclonal antibody IgG1k-neutralizing TNF- $\alpha$. Clinical trials as GO-REVEAL studies demonstrated a satisfactory efficacy of GLM in improving PsA signs and symptoms and in treating the structural damage caused by the disease [8]. Treatment recommendations underline the relevance of the burden of skin $\mathrm{PsO}$ in patients affected by PsA and skin manifestations need to be a relevant part of the decisionmaking process for PsA therapeutic approach [9]. However, the effectiveness evaluation of GLM in a real-life setting is a crucial issue, in particular for skin manifestation. To date, only a few unsponsored long-term studies reported the efficacy of GLM treatment in PsA patients and their treatment adherence and, to our knowledge, the GLM effectiveness according to patients characteristics and lines of treatment (i.e., 1st, 2nd, and 3rd or more lines) was rarely considered $[10,11]$. Moreover, limited data are available on the effectiveness and safety of GLM in patients affected by both PsO and PsA in bio-naïve and TNF-insufficient responders (TNFIR), in particular concerning skin sustained efficacy.

To address these knowledge gaps, the aims of this multicenter observational study were to evaluate the efficacy and the long-term treatment retention rate in patients affected by PsA with associated PsO treated with GLM in a real-life setting. Moreover, we aimed at evaluating the treatment retention rate based on patient characteristics, such as gender, body mass index (BMI), presence of comorbidities, smoking habit, and line of GLM treatment.

\section{Materials and methods}

\section{Patients}

A retrospective observational study was conducted on consecutive outpatients diagnosed with moderate-severe PsA treated with GLM between August 2011 and December 2020 attending the combined derma-rheuma clinic in the Rheumatology Units of the University of Padova, the University of Rome Tor Vergata, and the University of Naples Federico II. The end-points of the study were to determine efficacy on both joint and skin manifestations in PsA patients, the retention rate, and safety profile of a 4-year follow-up of GLM treatment. Inclusion criteria were the following: (1) age $>18$ years; (2) diagnosis of PsA $>6$ months in accordance with the Classification for Psoriatic Arthritis (CASPAR) criteria [12] and concomitant PsO; (3) the occurrence of peripheral arthritis (at least 1 active joint) and active $\mathrm{PsO}$ according to Psoriasis Area Severity Index (PASI) score [13]; (4) indication to start GLM treatment.

Data were collected at baseline (T0) and after 6 (T6), 12 (T12), 24 (T24), and 48 (T48) months of GLM treatment. PsO was clinically diagnosed by an experienced dermatologist (MT) and inflammatory bowel disease (IBD) was assessed as active or inactive based on the gastroenterologist (EC) (clinical and/or endoscopic) evaluation. Patients' comorbidities were evaluated in accordance with the classification of diseases outlined in the Charlson Comorbidity Index [14]. The presence of comorbidities and concomitant therapies were investigated (yes/no) at all the scheduled assessments by reviewing the patients' medical records. Metabolic syndrome (MetS) was investigated in accordance with internationally recognized standards $[14,15]$. Previous therapies with biologic disease-modifying antirheumatic drugs (bDMARDs), use of concomitant conventional synthetic DMARDs (csDMARDs) (methotrexate-MTX, leflunomide, sulfasalazine, and cyclosporine A), non-steroidal antiinflammatory drugs (NSAIDs), and corticosteroid therapy were registered at baseline and during the follow-up.

The comprehensive evaluation by clinical examination, made by experienced rheumatologists (MSC, FC and AO), included Bath Ankylosing Spondylitis disease activity index (BASDAI), Ankylosing Spondylitis Disease Activity Score-C-reactive protein based (ASDAS-CRP), evaluation of dactylitis (yes/no), enthesitis (yes/no), tender joint count (in 68 joints), swollen joint count (in 66 joints), and Disease Activity in Psoriatic Arthritis (DAPSA) score. Both the PASI and the assessment of psoriatic onychopathy (yes/no) were made by an experienced dermatologist. Patients were evaluated for the occurrence of erosions in peripheral joints by radiographic exams by three experienced rheumatologists (PC, MGL, and MT). Axial involvement was defined as the 
presence of radiographic axial involvement (at least unilateral grade 2 sacroiliitis and/or either marginal or paramarginal syndesmophytes in the cervical, thoracic, or lumbar spine on spinal radiographs) or by the presence of MRI sacroileitis [16]. Patient-reported outcomes (PROs) comprised pain visual analogue scale (pVAS) and global health $(\mathrm{GH})$ and health assessment questionnaire modified for spondyloarthritis (HAQ-S). Inflammatory markers as erythrocyte sedimentation rate (ESR) and CRP were registered. Metrological indexes such as height, weight, BMI, and abdominal circumference were evaluated. All the patients were treated with subcutaneously GLM at a dosage of $50 \mathrm{mg}$ or $100 \mathrm{mg}$ depending on the weight (100 mg was used when the patient's weight was $\geq 100 \mathrm{~kg}$ ) at week 0 and every 4 weeks thereafter, in accordance with the manufacturers' instructions [7].

The drug's safety was evaluated by assessing adverse events and via standard laboratory testing, the retention rate was estimated at $6,12,24$, and 48 months of treatment.

The study was approved by the local ethics committees of the institutions involved. Informed consent was obtained from all the patients before they were included in the study that was conducted in accordance with the ethical principles of the Declaration of Helsinki and consistent with good clinical practice guidelines.

\section{Statistical analysis}

To test the normality of data sets, the D' Agostino and Pearson omnibus test was used. Continuous data are presented as mean and standard deviation (SD) or median and interquartile range (IQR) when appropriate. For categorical variables, absolute and relative frequencies are reported. Continuous variables were compared using a paired $t$-test or a Wilcoxon signed-rank test for paired samples. Categorical variables were compared using the chi-squared test or Fisher's exact test when appropriate. The drug survival rates were analyzed using the Kaplan-Meier estimates. Drug survival rates were read from the Kaplan-Meier survival curves. Differences in drug survival between groups were analyzed using a log-rank (Mantel-Cox) test, by stratifying for sex, BMI, smoking habit, and line of treatment. A $p$ value $<0.05$ was considered statistically significant. All statistical analyses were performed using GraphPad Prism version 7 (GraphPad software).

\section{Results}

\section{Patient characteristics}

Data from a total of 105 patients affected by moderate to severe PsA who started treatment with GLM were registered. Baseline clinical and laboratory characteristics are summarized in Table 1.
Peripheral arthritis was present in $68(64.8 \%)$ cases and axial disease in 37 cases (35.2\%). In particular, oligoarthritis was denoted in 30 cases (28.6\%) and polyarthritis in 75 cases (71.4\%). In the group of patients with axial disease, singlejoint arthritis occurred in 17 patients (45.9\%), oligoarthritis in 6 patients (16.2\%), and polyarthritis in 10 cases (27\%). No cases of arthritis mutilans or prominent distal interphalangeal joint (DIP) involvement were registered. Enthesitis and $\mathrm{PsO}$ were described in $82(78 \%)$ and $105(100 \%)$ patients, respectively. Moreover, peri-articular manifestations were reported in 36 (34.2\%) patients. Extra-articular manifestations including IBD were observed in $20(19 \%)$ patients (13 with inactive IBD and 7 patients with active disease) while isolated anterior uveitis occurred in a single case $(0.9 \%)$.

The most frequent comorbidity described was the occurrence of cardiovascular diseases in 33 (31.4\%) patients and MetS in 20 (19\%) patients. A relevant incidence of smokers (33 patients, $31.4 \%$ ) was registered as well as an elevated mean BMI score (27.1 \pm 6.0$)$, with overweight in 39 (37.1\%) cases and a defined obesity in $20(19 \%)$ cases.

\section{Golimumab effectiveness}

According to the primary end-point of the study, a statistical significant improvement of the DAPSA score was observed at T6, T12, T24, T36, and T48 months $(p<0.0001$ for all comparisons, Fig. 1A). ASDAS-CRP levels significantly reduced at all time points $(p<0.0001$ at T6, $p<0.0001$ at $\mathrm{T} 12, p=0.0001$ at $\mathrm{T} 24, p=0.0005$ at $\mathrm{T} 36$, and $p=0.0002$ at T48, Fig. 1B) while BASDAI score reduced at T6 $(p<0.0001), \mathrm{T} 12(p<0.0001), \mathrm{T} 24(p=0.0002)$, T36 ( $p=0.004)$, and T48 $(p=0.002)$ (Fig. 1C). A significantly reduction in the frequency of dactylitis was observed at T6 $(n=9,8.6 \%), \mathrm{T} 12(n=2,1.9 \%), \mathrm{T} 24(n=2,1.9 \%)$, T36 $(n=0,0 \%)$, and T48 $(n=0,0 \%)$. This data was also confirmed for enthesitis at T6 $(n=52,49.5 \%), \mathrm{T} 12(n=16$, $15.2 \%), \mathrm{T} 24(n=7,6.6 \%), \mathrm{T} 36(n=2,1.9 \%)$, and T48 $(n=2,1.9 \%)$. A substantial improvement was also detected in skin involvement: PASI score at T6 $(p<0.0001)$, T12 $(p<0.0001), \mathrm{T} 24(p=0.001)$, and T36 and T48 $(p<0.0001$ for both comparisons) (Fig. 1D).

CRP levels decreased at T6, T12, T24 ( $p<0.0001$ for all comparisons); T36 ( $p=0.0009)$; and T48 $(p=0.0007)$ (Fig. 2A). GH and pain were reduced at all the time points ( $p<0.0001$ for all comparisons), and disability improved at T6 $(p<0.0001)$, T12 $(p<0.0001)$, T24 $(p<0.0001)$, T36 $(p=0.0005)$, and T48 ( $p=0.0002)$ (Fig. 2B-D). Clinical improvement was also evaluated by the gain of DAPSA low disease activity: at T6 $(n=54) 62.1 \%$, T12 $(n=49) 63.6 \%$, T24 $(n=36) 55.4 \%, \mathrm{~T} 36(n=35,61.5 \%)$, and T48 $(n=35$, 61.5\%) and DAPSA remission score: at T6 $(n=11) 12.6 \%$, $\mathrm{T} 12(n=16) 20.8 \%, \mathrm{~T} 24(n=17) 26.2 \%$, T36 $(n=21) 36.8 \%$, and T48 $(n=21) 36.8 \%$. 
Table 1 Baseline clinical and serological characteristics of patients

\begin{tabular}{ll}
\hline$N$ & 105 \\
\hline Age, years & $53.04 \pm 11.6$ \\
M/F & $44 / 61$ \\
Disease duration, months & $174.9 \pm 93.0$ \\
PsO, $n$ (\%) & $105(100 \%)$ \\
Peripheral disease, $n(\%)$ & $68(64.7 \%)$ \\
Tender joint count & $8.1 \pm 5.5$ \\
Swollen joint count & $2.7 \pm 3.1$ \\
Axial disease, $n(\%)$ & $37(35.3 \%)$ \\
Enthesitis, $n(\%)$ & $82(78 \%)$ \\
Dactylitis, $n(\%)$ & $36(34.2 \%)$ \\
ESR, mm/h & $24.1 \pm 18.9$ \\
CRP, mg/dL & $1.2 \pm 2.3$ \\
DAPSA & $25.7 \pm 9.7$ \\
BASDAI & $6.8 \pm 2.5$ \\
PASI & $2.3 \pm 3.5$ \\
ASDAS-CRP & $3.3 \pm 0.8$ \\
Active IBD & $7(6.6 \%)$ \\
Crohn disease, $n(\%)$ & $2(1.9 \%)$ \\
RCU disease, $n(\%)$ & $5(4.8 \%)$ \\
Inactive IBD, $n(\%)$ & $13(12.4 \%)$ \\
Inactive uveitis, $n(\%)$ & $1(0.9 \%)$ \\
BMI & $27.1 \pm 6.0$ \\
HLA-B27 positive, $n(\%)$ & $11(10.5 \%)$ \\
Smoking, $n(\%)$ & $33(31.4 \%)$ \\
Cardiovascular comorbidities, $n(\%)$ & $33(31.4 \%)$ \\
Metabolic comorbidities, $n(\%)$ & $20(19 \%)$ \\
Hashimoto's disease, $n(\%)$ & $7(6.7 \%)$ \\
Concomitant csDMARD, $n(\%)$ & $60(57.1 \%)$ \\
Concomitant glucocorticoids, $n(\%)$ & $40(38.1 \%)$ \\
Concomitant NSAIDs, $n(\%)$ & $42(40 \%)$ \\
Biologic-naïve, $n(\%)$ & $44(41.9 \%)$ \\
GLM as second line, $n(\%)$ & $25(23.8 \%)$ \\
GLM as third line, $n(\%)$ & $27(25.7 \%)$ \\
GLM as fourth line or more, $n(\%)$ & $9(8.6 \%)$ \\
\hline &
\end{tabular}

Data are expressed as mean \pm standard deviation

$M$ male, $F$ female, $P s O$ psoriasis, GLM golimumab, NSAIDs nonsteroidal anti-inflammatory drugs, $B M I$ body mass index, $c s D M A R D$ conventional synthetic disease-modifying antirheumatic drugs, ESR erythrocyte sedimentation rate, CRP C-reactive protein, DAPSA disease activity in psoriatic arthritis, BASDAI Bath Ankylosing Spondylitis disease activity index, ASDAS-CRP, Ankylosing Spondylitis Disease Activity Score-C-reactive protein based, IBD inflammatory bowel diseases, PASI Psoriasis Area Severity Index

\section{Drug survival and safety}

In order to explore the clinical response, survival rate curves at $6,12,24$, and 48 months, as well as the reasons for treatment discontinuation, were assessed.

Figure 3A shows the GLM retention rate estimated by the Kaplan-Meier analysis. After 6 months of treatment, the retention rate was $82.8 \%$, at 12 months $73.4 \%$, at 24 months $62 \%$, and at 48 months $54.4 \%$. No statistically significant difference emerged when patients were divided according to gender ( $p=0.652$ ) (Fig. 3B). Similarly, the division of patients into 3 categories based on their BMI (normal weight with $\mathrm{BMI}<25$, overweight with $\mathrm{BMI} \geq 25$, and obesity with $\mathrm{BMI} \geq 30$ ) did not conduce to any statistically significant result ( $p=0.655$ ) (Fig. 3C). Data were analyzed by distinguishing patients based on smoking habits and the occurrence of comorbidities. In relation to smoking, no difference was found between smokers and non-smokers $(p=0.466)$ (Fig. 3D). With regard to the presence of comorbidities, no differences were noted between groups $(p=0.902)$ (Fig. 4A). On the other hand, the analysis of the three distinct subgroups of comorbidities highlighted that the presence of a thyroid disease condition was a negative prognostic factor in treatment persistence ( $p=0.030$ ) (Fig. 4B-D), while no statistically significant correlation was observed between the presence of MetS and persistence in therapy $(p=0.238)$ and between gastro-enteric pathologies and persistence in therapy $(p=0.867)$.

Finally, data were analyzed by focusing on lines of treatment and association with MTX. Concerning lines of treatment, patients were divided into biological naïve and patients who had previously taken other biologicals: there were no statistically significant differences between these two study populations $(p=0.208)$. Based on the association or not with MTX, considering valid the combination therapy only if carried out for a period of time equal to or greater than half of the total duration of follow-up: no difference was found in patients treated as monotherapy compared with those taking MTX $(p=0.599)$.

Overall, in the follow-up, reasons for drug discontinuation were primary inefficacy in 13 cases, secondary inefficacy in 21 cases, and 5 cases were lost at follow-up. For the event, reasons for discontinuation were mild infections in 5 cases and surgery in 1 case not related to the treatment.

\section{Discussion}

In this observational retrospective, multicenter study in patients with moderate to severe PsA with cutaneous manifestations of PsO, GLM was effective for both articular and skin involvement. Indeed, GLM was effective in reducing all clinical parameters analyzed at all the time points of evaluation until 48 months of observation including inflammatory indexes and pain. Data at 36 months were not shown because stackable with those at 48 months. Clinical phenotype of patients was characterized by cutaneous involvement represented in all patients while, concerning articular and peri-articular manifestations, a high prevalence of polyarticular involvement and enthesitis was found. Long-standing 
Fig. 1 A-D Golimumab effectiveness measured by composite clinimetric indexes and PASI score. $* * p<0.01$; $* * * p<0.001 ; * * * * p<0.0001$. DAPSA Disease Activity in Psoriatic Arthritis, BASDAI Bath Ankylosing Spondylitis disease activity index, $A S D A S-C R P$ Ankylosing Spondylitis Disease Activity Score-C-reactive protein based, PASI Psoriasis Area Severity Index

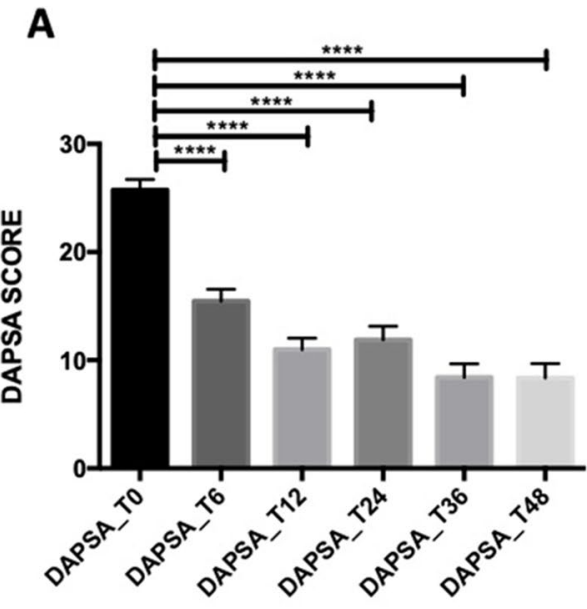

C

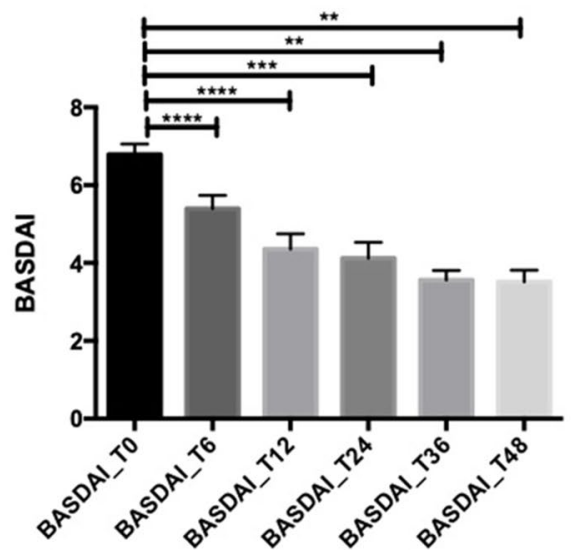

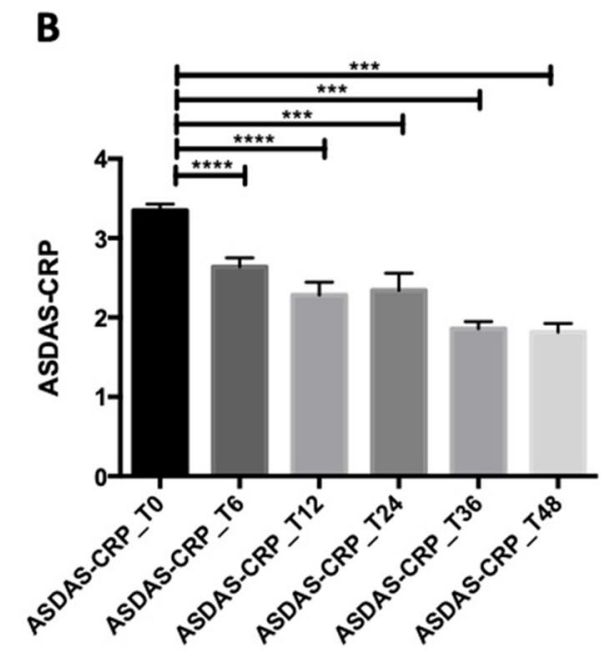

D

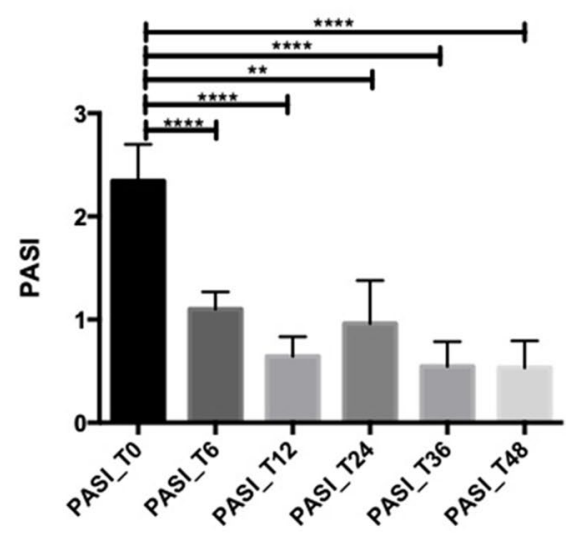

disease, high prevalence of erosions, and presence of axial disease demonstrated the presence of a challenging study population of PsA patients. Moreover, the high disease activity at baseline measured by specific composite scores supported the severity of PsA. The statistically significant reduction of clinimetrics indexes, inflammatory markers, and the gain of a high percentage of patients in DAPSA low disease activity support the efficacy of GLM in our "difficult-totreat" PsA patients. Remission according to DASPA score, an ambitious endpoint, was obtained in $20 \%$ of patients at $\mathrm{T} 12$ and $36.8 \%$ of patients were in clinical remission at $\mathrm{T} 48$. In this context, we assume that TNF inhibition represents one of the key treatments for PsA and PsO, as confirmed by clinical trials demonstrating the efficacy of TNFi in both PsA and $\mathrm{PsO}$ [5]. The reduction of inflammatory markers, such as $\mathrm{CRP}$, considered a biomarker of radiographic progression in $\mathrm{SpA}$, may be considered as an indirect outcome of the reduction of radiographic damage [17]. Based on ASDAS-CPR and BASDAI scores, significant improvement was reached and maintained within the first 12 months of treatment and sustained during the following 48 months of follow-up for axial involvement. Most patients evaluated in our study had been previously treated with other biologic agents (TNFi) and only $41.9 \%$ of patients were bio-naïve with $57 \%$ in concomitant csDMARD treatment. The drug survival of GLM was independent of the line of treatment used and the previous use of bDMARDs and can be considered effective both as first-line therapy and in patients with failure for one or more bDMARDs. Data from international and national registries, such as the GISEA registry or the BioRx.si, have compared the characteristics of patients starting treatment with GLM [11, 18, 19]. bDMARD-naïve and bDMARDexperienced patients were compared and no difference in the persistence of GLM was found. The switch among the same group of drugs, as the TNFi family, is still a debate in PsA management and patients failing a first TNFi agent usually use a different target [11]. However, several data from the literature seem to support the efficacy of switching among TNFi in PsA $[10,19]$. The evaluation of drug persistence in our real-life experience demonstrated a good percentage (54\%) of patients still on therapy after 4 years of GLM treatment. Other studies, evaluating the same outcome, estimated 
Fig. 2 A-D Golimumab effectiveness measured by patientreported outcomes and inflammatory markers. $* * p<0.01$; $* * * p<0.001 ; * * * * p<0.0001$. $C R P$ C-reactive protein, $G H$ global health, $p V A S$ pain-visual analogue scale, $H A Q$ health assessment questionnaire

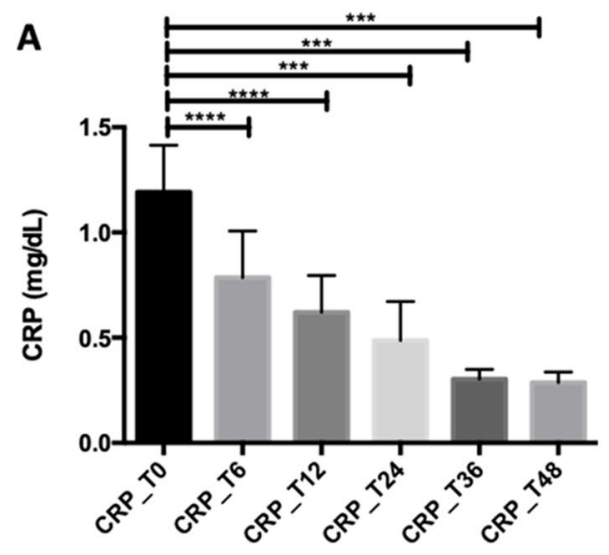

B

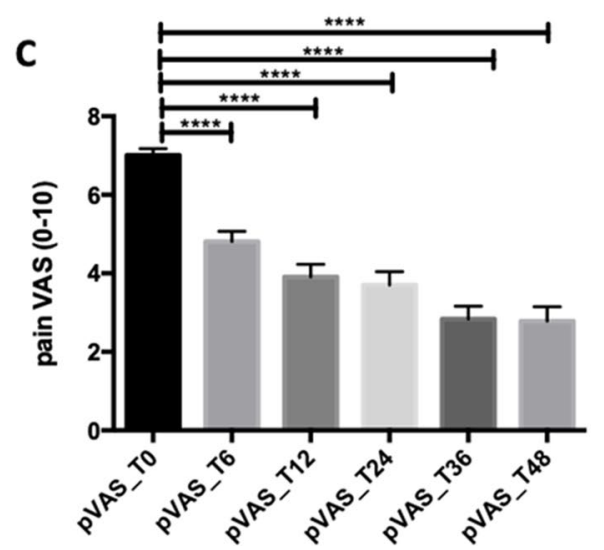

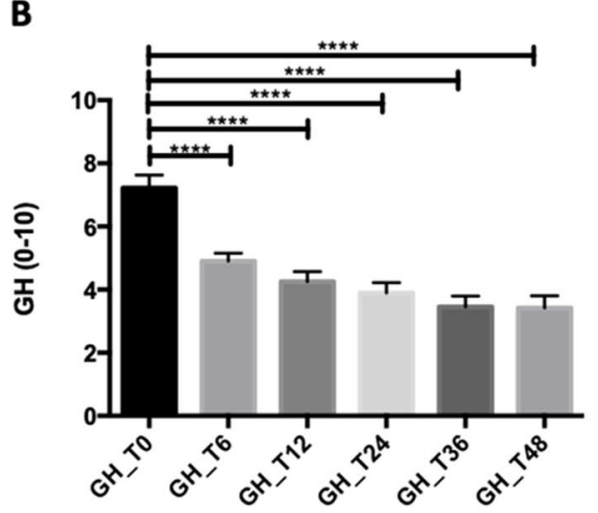

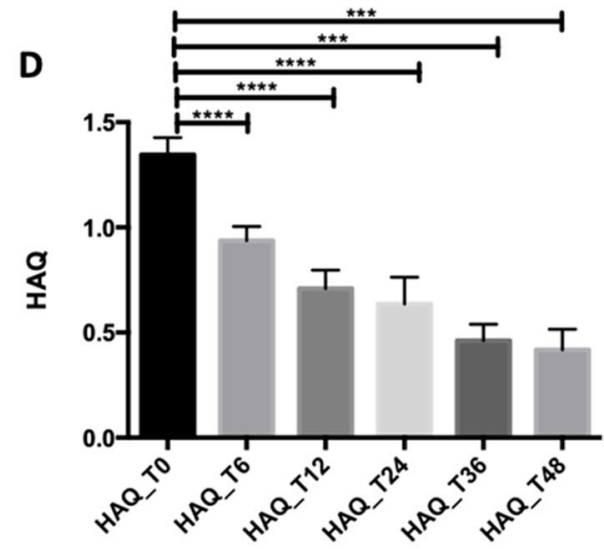

approximately $47 \%$ of PsA patients, from the UK, remained on their initial TNFi therapy after 5 years and low persistence of $32 \%$ after 5 years of follow-up on PsA patients in Germany, while no differences among TNFi were observed [20-22].

Recently, none of the TNFi agents was found to be more persistent than others as first-line therapy, while secukinumab was found to be superior to other biologics when indicated as second-line therapy [23]. However, data from Slovenia showed a better persistence of GLM compared to other TNFis in bDMARD-experienced AS and PsA patients [19].

The use of combination therapy as bDMARDs with MTX or other csDMARDs is still controversial in the management of PsA since its rational use is based on indirect evidence or expert opinion [24]. However, csDMARDs may be warranted in peripheral joint manifestations when bDMARDs are not effective [25]. We have not observed significant differences in survival rates among patients using MTX or not, confirming that GLM was effective in both combination treatment and monotherapy. This result suggests to using the GLM also in those patients that are intolerant to csDMARDs, making MTX treatment not necessary when a bDMARD is started. Furthermore, no difference in survival rates was observed when patients were divided according to the presence of comorbidities, BMI, or gender.
MetS was strongly associated with a lower probability to achieve MDA in TNFi-treated PsA patients, and different studies reported a higher prevalence of MetS in PsA patients compared to other rheumatic diseases [26, 27]. In our cohort of patients, MetS was not a negative predictor factor for GLM efficacy, in contrast with other findings supporting the hypothesis that MetS or overweight and obesity may affect TNFi therapy, as adalimumab or etanercept. GLM efficacy might not be affected by the presence of MetS because the dosing regimen is adequate to the weight of the patients being MetS related to chronic inflammatory state and adipokines. Lifestyle such as smoking habit is associated with high disease activity in PsA patients and may exert a negative role during the treatment with bDMARDs [28]. We have not observed the influence of smoking in our 4 years of follow-up, making GLM effective also in smokers.

Among other factors affecting treatment response, gender may influence bDMARDs clinical response remaining a challenge in PsA. In particular, female gender has been associated with a prevalent polyarticular phenotype, high swollen joint count, and peripheral erosive joint involvement compared to males, while female gender correlated with lower rates of extraarticular manifestations and axial involvement [28, 29]. Moreover, the female gender generally associates with poor rates of response to TNFi and a lower probability of achieving 

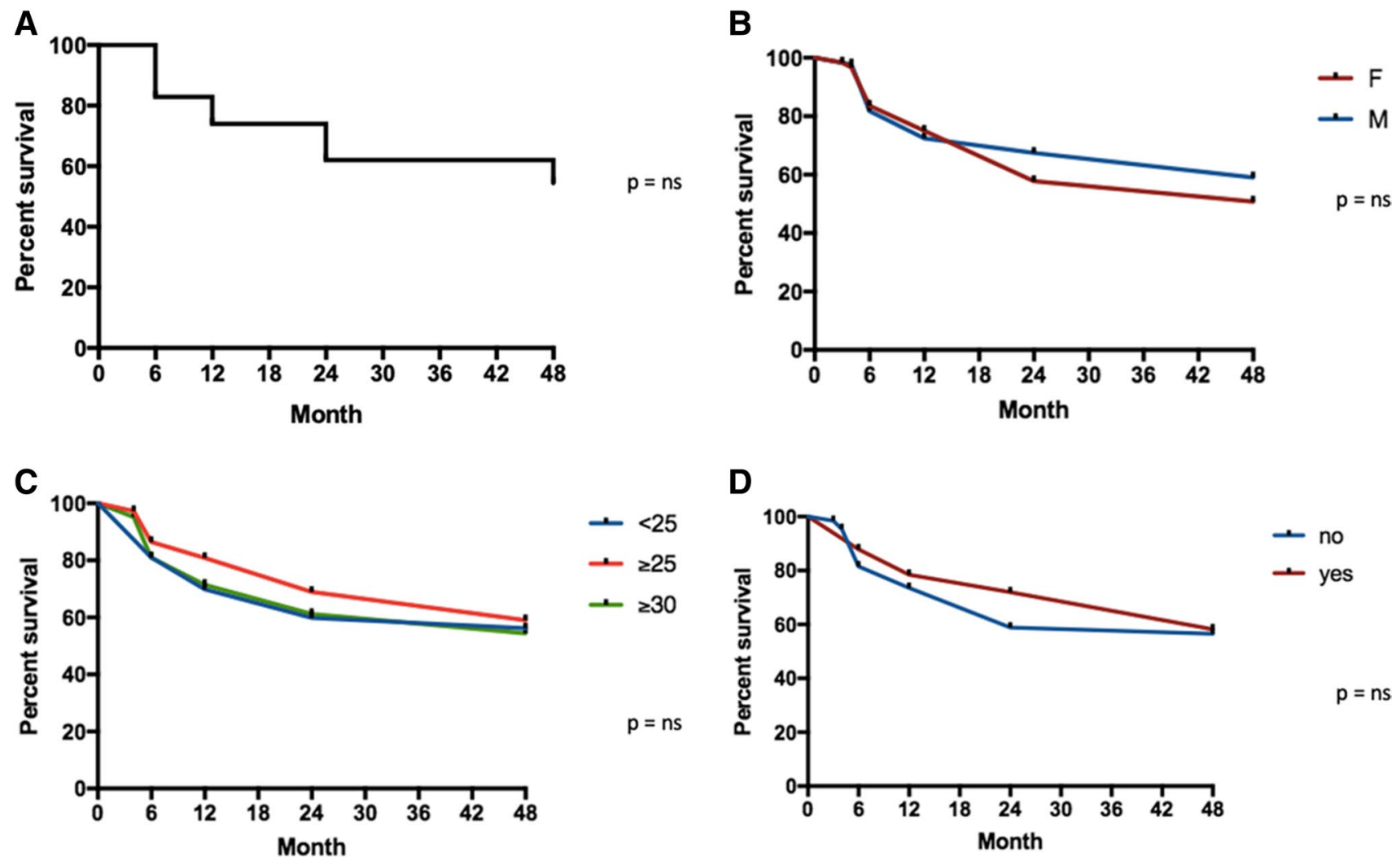

Fig. 3 A-D. Forty-eight-month retention rate global (A) and according to gender (B), BMI (C), and smoke habit (D). $M$ male, $F$ female; $<25$ normal weight, $>25$ overweight, $>30$ obese

A

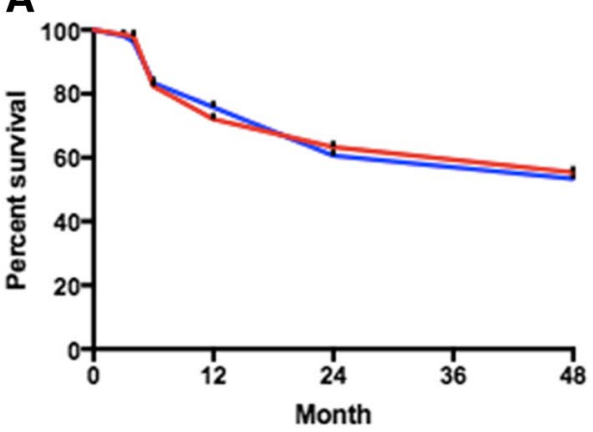

C

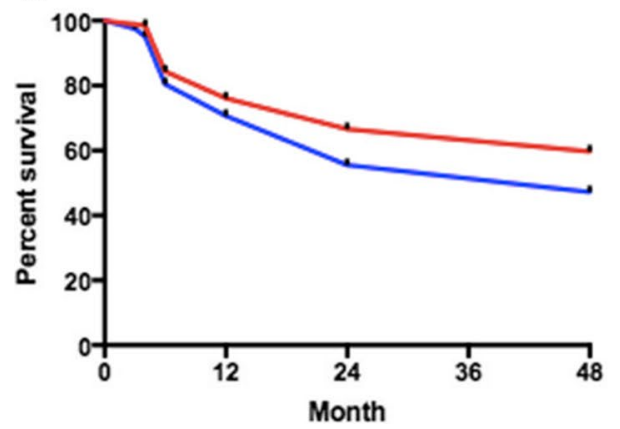

B
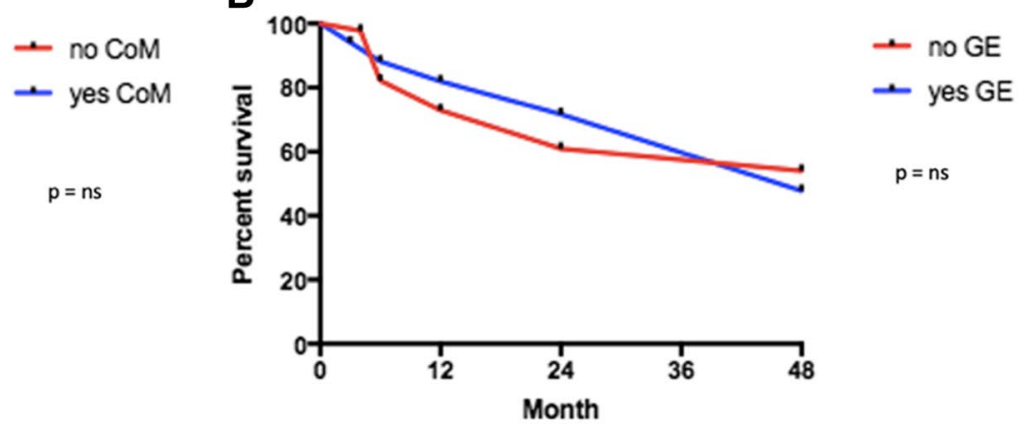

D

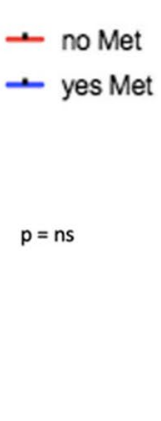

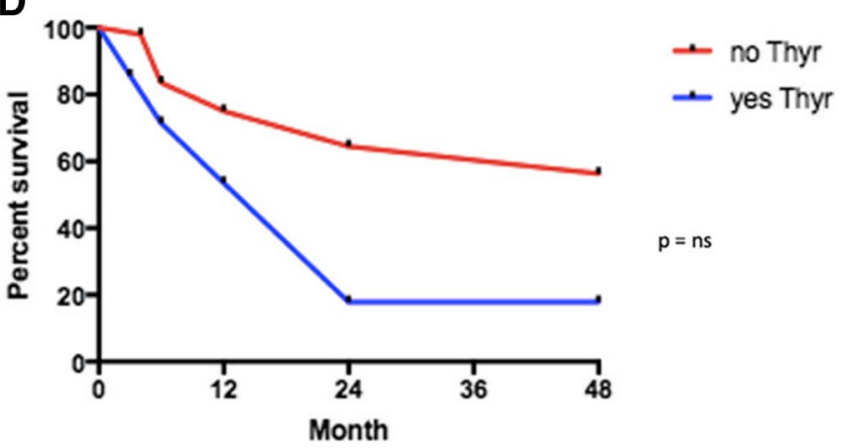

Fig. 4 A-D Forty-eight-month retention rate according to comorbidities (A), gastro-enteric pathologies (B), metabolic dysfunctions (C), and thyroid disease (D). CoM comorbidities, GE gastro-enteric, Met metabolic dysfunctions, Thyr thyroid disease 
remission compared to males [30], especially when comorbidities, such as MetS, occur [30-32]. In our cohort, we did not observe gender differences in the efficacy of GLM, since gender was not a relevant factor in patient outcomes. The high prevalence of IBD in our cohort is due to the early referral in the combined gastro-rheuma outpatient clinic by gastroenterologists from the involved hospitals $[33,34]$. Thyroid dysfunction may be associated with the worst clinical outcome in several inflammatory conditions and in PsA $[26,35,36]$. According to our data, thyroid disorders may represent a negative prognostic factor in the PsA study population for treatment persistence even if this result needs to be confirmed in a larger study cohort. Thyroid disorders may have a relevant impact on disease perception and activity and so may have an influence on treatment efficacy.

A very low rate of primary and secondary failure was observed with a good safety profile with no serious adverse events reported consistent with previous reports of patients treated with GLM [37-39].

This study encompasses limitations such as the retrospective nature of the study, the presence of low cutaneous disease activity at baseline, the relatively low number of PsA patients enrolled due to the inclusion of patients with both joint and skin manifestations, and the absence of imaging in the follow-up (radiographic evaluation of efficacy), even if ultrasound, MRI, and/or X-ray was performed in all patients to confirm the diagnosis at baseline.

\section{Conclusion}

In this multicentric study, we described the clinical efficacy in joint and periarticular structures as well as on PsO manifestations on moderate to severe PsA patients treated with GLM in a real-life setting. Treatment efficacy was supported by a good drug persistence of GLM regardless of the presence of comorbidities and patients' characteristics. Our results were consistent with no differences in terms of clinical response and efficacy between males and females, smokers and no-smokers, obese and normal-weight patients, and lines of treatment, confirming GLM efficacy and safety in a long-term real-life setting.

Acknowledgements The authors are grateful to Dr. Miriam Teoli for the dermatological assessment and Dr. Emma Calabrese for the gastroenterologic evaluation of patients, and Dr. Laura Mathew for the revision of the English language.

Funding Open access funding provided by Università degli Studi di Roma Tor Vergata within the CRUI-CARE Agreement.

\section{Declarations}

Conflict of interest All the authors declared that they have no conflict of interest.
Open Access This article is licensed under a Creative Commons Attribution 4.0 International License, which permits use, sharing, adaptation, distribution and reproduction in any medium or format, as long as you give appropriate credit to the original author(s) and the source, provide a link to the Creative Commons licence, and indicate if changes were made. The images or other third party material in this article are included in the article's Creative Commons licence, unless indicated otherwise in a credit line to the material. If material is not included in the article's Creative Commons licence and your intended use is not permitted by statutory regulation or exceeds the permitted use, you will need to obtain permission directly from the copyright holder. To view a copy of this licence, visit http://creativecommons.org/licenses/by/4.0/.

\section{References}

1. Calabresi E, Monti S, Terenzi R, Zanframundo G, Perniola S, Carli L (2020) One year in review 2019: psoriatic arthritis. Clin Exp Rheumatol 38:1046-1055

2. Chimenti MS, Esposito M, Graceffa D, Teoli M, Peluso G, Birra D et al (2019) PsA-Disk, a novel visual instrument to evaluate psoriatic arthritis in psoriatic patients: an Italian derma-rheuma multicentre study. Ther Adv Chronic Dis 10:2040622319847056. https://doi.org/10.1177/2040622319847056

3. Lubrano E, Delle Sedie A, Romanelli M, Chimenti MS, Bianchi L, Piaserico $S$ et al (2019) Management of psoriatic arthritis in rheumatology and dermatology settings: sub-analysis of the Italian population from the international LOOP study. Clin Rheumatol. https://doi.org/10.1007/s10067-020-05482

4. Chimenti MS, Triggianese P, De Martino E, Conigliaro P, Fonti GL, Sunzini F et al (2019) An update on pathogenesis of psoriatic arthritis and potential therapeutic targets. Expert Rev Clin Immunol 15:823-836. https://doi.org/10.1080/1744666X.2019.1627876

5. Chimenti MS, D'Antonio A, Conigliaro P, Ferrigno S, Vendola A, Ferraioli M, Triggianese P, Costa L, Caso F, Perricone R (2020) An update for the clinician on biologics for the treatment of psoriatic arthritis. Biologics 14:53-75. https://doi.org/10.2147/BTT. S260754

6. D'Angelo S, Cantini F, Ramonda R, Cantarini L, Carletto A, Chimenti MS et al (2019) Effectiveness of adalimumab for the treatment of psoriatic arthritis: an Italian real-life retrospective study. Front Pharmacol 10:1497. https://doi.org/10.3389/fphar. 2019.01497

7. https://www.ema.europa.eu/en/medicines/human/EPAR/simponi

8. Kavanaugh A, McInnes IB, Mease P, Krueger GG, Gladman D, van der Heijde D, Zhou Y, Lu J, Leu JH, Goldstein N, Beutler A (2014) Clinical efficacy, radiographic and safety findings through 5 years of subcutaneous golimumab treatment in patients with active psoriatic arthritis: results from a long-term extension of a randomised, placebo-controlled trial (the GO-REVEAL study). Ann Rheum Dis 73:1689-1694. https://doi.org/10.1136/annrh eumdis-2013-204902

9. Coates LC, Kavanaugh A, Mease PJ, Soriano ER, AcostaFelquer ML, Armstrong AW et al (2016) Group for Research and Assessment of Psoriasis and Psoriatic Arthritis 2015 treatment recommendations for psoriatic arthritis. Arthritis Rheumatol 68:1060-1071. https://doi.org/10.1002/art.39573

10. Chimenti MS, Teoli M, Saraceno R, Dattola A, Ventura A, Chiricozzi A, Chiaramonte C, Perricone R, Chimenti S (2013) Golimumab in patients affected by moderate to severe psoriatic arthritis: an open-label study in thirty-two patients previously 
treated with other biologics. Dermatology 227:305-310. https:// doi.org/10.1159/000354263

11. Iannone F, Favalli EG, Caporali R, D'Angelo S, Cantatore FP, Sarzi-Puttini P et al (2020) Golimumab effectiveness in biologic inadequate responding patients with rheumatoid arthritis, psoriatic arthritis and spondyloarthritis in real-life from the Italian registry GISEA. Joint Bone Spine 88:105062. https://doi.org/ 10.1016/j.jbspin.2020.07.011

12. Taylor W, Gladman D, Helliwell P, Marchesoni A, Mease P, Mielants H (2006) Classification criteria for psoriatic arthritis: development of new criteria from a large international study. Arthritis Rheum 54:2665-73. https://doi.org/10.1002/ art.21972

13. Gisondi P, Altomare G, Ayala F, Bardazzi F, Bianchi L, Chiricozzi A et al (2017) Italian guidelines on the systemic treatments of moderate-to-severe plaque psoriasis. J Eur Acad Dermatol Venereol 31:774-790. https://doi.org/10.1111/jdv.14114

14. Charlson ME, Pompei P, Ales KL, MacKenzie CR (1987) A new method of classifying prognostic comorbidity in longitudinal studies: development and validation. J Chronic Dis 40:373-383. https://doi.org/10.1016/0021-9681(87)90171-8

15. Alberti KG, Eckel RH, Grundy SM et al (2009) Harmonizing the metabolic syndrome: a joint interim statement of the International Diabetes Federation Task Force on Epidemiology and Prevention; National Heart, Lung, and Blood Institute; American Heart Association; World Heart Federation; International Atherosclerosis Society; and International Association for the Study of Obesity. Circulation 120:1640-1645. https://doi.org/ 10.1161/CIRCULATIONAHA.109.192644

16. Feld J, Ye JY, Chandran V et al (2021) Axial disease in psoriatic arthritis: the presence and progression of unilateral grade 2 sacroiliitis in a psoriatic arthritis cohort. Semin Arthritis Rheum 51:464-468. https://doi.org/10.1016/j.semarthrit.2021.03.007

17. Maksymowych WP (2019) Biomarkers for diagnosis of axial spondyloarthritis, disease activity, prognosis, and prediction of response to therapy. Front Immunol 10:305. https://doi.org/10. 3389/fimmu.2019.00305

18. Rotar Ž, Tomšič M, Praprotnik S, Rheumatologists S (2019) The persistence of golimumab compared to other tumour necrosis factor- $\alpha$ inhibitors in daily clinical practice for the treatment of rheumatoid arthritis, ankylosing spondylitis and psoriatic arthritis: observations from the Slovenian nation-wide longitudinal registry of patients treated with biologic disease-modifying antirheumatic drugs-BioRx.si. Clin Rheumatol 38:297-305. https://doi.org/10.1007/s10067-018-4324-7

19. Alegre-Sancho JJ, Juanola X, Rodríguez-Heredia JM, Manero J, Villa-Blanco I, Laiz A, Arteaga MJ, Cea-Calvo L, González CM (2021) Effectiveness and persistence of golimumab as a second biological drug in patients with spondyloarthritis: a retrospective study. Medicine (Baltimore) 100:e25223. https://doi.org/ 10.1097/MD.0000000000025223

20. Saad AA, Ashcroft DM, Watson KD, Hyrich KL, Noyce PR, Symmons DPM et al (2009) Persistence with anti-tumour necrosis factor therapies in patients with psoriatic arthritis: observational study from the British Society of Rheumatology Biologics Register. Arthritis Res Ther 11:R52

21. Jacob L, Chevalier T, Kostev K (2019) Persistence with biological drugs in patients treated in rheumatology practices in Germany. Rheumatol Int 39(3):525-531

22. Costa L, Perricone C, Chimenti MS, Del Puente A, Caso P, Peluso $\mathrm{R}$ et al (2017) Switching between biological treatments in psoriatic arthritis: a review of the evidence. Drugs R D 17:509-522
23. Haddad A, Gazitt T, Feldhamer I et al (2021) Treatment persistence of biologics among patients with psoriatic arthritis. Arthritis Res Ther 23:44. https://doi.org/10.1186/s13075-021-02417-x

24. Gossec L, Baraliakos X, Kerschbaumer A, de Wit M, McInnes I, Dougados M et al (2020) EULAR recommendations for the management of psoriatic arthritis with pharmacological therapies: 2019 update. Ann Rheum Dis 79:700-712. https://doi.org/ 10.1136/annrheumdis-2020-217159

25. Heiberg MS, Kaufmann C, Rødevand E, Mikkelsen K, Koldingsnes W, Mowinckel P, Kvien TK (2007) The comparative effectiveness of anti-TNF therapy and methotrexate in patients with psoriatic arthritis: 6 month results from a longitudinal, observational, multicentre study. Ann Rheum Dis 66:1038-1042. https:// doi.org/10.1136/ard.2006.064808

26. Chimenti MS, Triggianese P, Conigliaro P, Tonelli M, Gigliucci G, Novelli L, Teoli M, Perricone R (2017) A 2-year observational study on treatment targets in psoriatic arthritis patients treated with TNF inhibitors. Clin Rheumatol 36:2253-2260. https://doi. org/10.1007/s10067-017-3769-4

27. Costa L, Caso F, Ramonda R, Del Puente A, Cantarini L, Darda MA, Caso P, Lorenzin M, Fiocco U, Punzi L, Scarpa R (2015) Metabolic syndrome and its relationship with the achievement of minimal disease activity state in psoriatic arthritis patients: an observational study. Immunol Res 61:147-153. https://doi.org/ 10.1007/s12026-014-8595-z

28. Chimenti MS, Fonti GL, Conigliaro P, Sunzini F, Scrivo R, Navarini $L$ et al (2020) One-year effectiveness, retention rate, and safety of secukinumab in ankylosing spondylitis and psoriatic arthritis: a real-life multicenter study. Expert Opin Biol Ther 20:813-821. https://doi.org/10.1080/14712598.2020.1761957

29. Eder L, Chandran V, Gladman DD (2012) Gender-related differences in patients with psoriatic arthritis. Int J Clin Rheumatol 7:641-649

30. Queiro R, Sarasqueta C, Torre JC, Tinture T, Lopez-Lagunas I (2001) Comparative analysis of psoriatic spondyloarthropathy between men and women. Rheumatol Int 21:66-68. https://doi. org/10.1007/s002960100135

31. Wallenius M, Skomsvoll JF, Koldingsnes W, Rodevand E, Mikkelsen K, Kaufmann C et al (2009) Work disability and healthrelated quality of life in males and females with psoriatic arthritis. Ann Rheum Dis 68:685-689. https://doi.org/10.1136/ard.2008. 092049

32. Duruöz MT, Gezer HH, Nas K, Kılıç E, Sargın B, Kasman SA et al (2021) Gender-related differences in disease activity and clinical features in patients with peripheral psoriatic arthritis: a multi-center study. Joint Bone Spine 88:105177. https://doi.org/ 10.1016/j.jbspin.2021.105177

33. Conigliaro P, Chimenti MS, Ascolani M et al (2016) Impact of a multidisciplinary approach in enteropathic spondyloarthritis patients. Autoimmun Rev 15:184-190

34. Conigliaro P, Chimenti MS, Triggianese P et al (2021) Two years follow-up of golimumab treatment in refractory enteropathic spondyloarthritis patients with Crohn disease: a STROBE-compliant study. Medicine (Baltimore) 100:e25122. https://doi.org/ 10.1097/MD.0000000000025122

35. Triggianese $\mathrm{P}$, Perricone $\mathrm{C}$, Conigliaro $\mathrm{P}$, Chimenti MS, Perricone R, De Carolis C (2016) Peripheral blood natural killer cells and mild thyroid abnormalities in women with reproductive failure. Int J Immunopathol Pharmacol 29:65-75

36. Conigliaro P, D'Antonio A, Pinto S et al (2020) Autoimmune thyroid disorders and rheumatoid arthritis: a bidirectional interplay. Autoimmun Rev 19:102529. https://doi.org/10.1016/j.autrev.2020. 102529 
37. Manara M, Caporali R, Favalli EG, Grosso V, Atzeni F, Sarzi Puttini P et al (2017) Two-year retention rate of golimumab in rheumatoid arthritis, psoriatic arthritis and ankylosing spondylitis: data from the LORHEN registry. Clin Exp Rheumatol 35:804-809

38. Iannone F, Santo L, Anelli MG, Bucci R, Semeraro A, Quarta L et al (2017) Golimumab in real-life settings: 2 years drug survival and predictors of clinical outcomes in rheumatoid arthritis, spondyloarthritis, and psoriatic arthritis. Semin Arthritis Rheum 47:108-114. https://doi.org/10.1016/j.semarthrit.2017.01.008
39. Pombo-Suarez M, Sanchez-Piedra C, Garcia-Magallón B, PérezGómez A, Manrique-Arija S, Martín-Doménech R et al (2021) Factors associated with long-term retention of treatment with golimumab in rheumatoid arthritis, axial spondyloarthritis, and psoriatic arthritis: an analysis of the Spanish BIOBADASER registry. Clin Rheumatol. https://doi.org/10.1007/s10067-021-05742-3

Publisher's note Springer Nature remains neutral with regard to jurisdictional claims in published maps and institutional affiliations. 